\title{
Management of a Compressive Goiter at Term
}

\author{
Hanne De Mulder ${ }^{1}$, Kieran Dempster-Rivett ${ }^{2}$ \\ ${ }^{1}$ Department of Obstetrics and Gynecology, UZ Leuven, Leuven, Belgium \\ ${ }^{2}$ Department of Obstetrics and Gynecology, Middlemore Hospital, Auckland, New Zealand \\ Email: hanne.demulder@student.kuleuven.be
}

How to cite this paper: De Mulder, $\mathrm{H}$. and Dempster-Rivett, K. (2021) Management of a Compressive Goiter at Term. Open Journal of Obstetrics and Gynecology, 11, 303308.

https://doi.org/10.4236/ojog.2021.113030

Received: January 25, 2021

Accepted: March 19, 2021

Published: March 22, 2021

Copyright $\odot 2021$ by author(s) and Scientific Research Publishing Inc. This work is licensed under the Creative Commons Attribution International License (CC BY 4.0).

http://creativecommons.org/licenses/by/4.0/

\begin{abstract}
Pregnant women sometimes have thyroid disease, and thus goiter; however, it does not become so large to the extent that the goiter obstructs the air way, causing respiratory symptoms. Here, we report such a case. A 27-year-old woman with large goiter presented with life threatening stridor at 37 weeks. After a multidisciplinary-team discussion, we performed caesarean section plus thyroidectomy, which resulted in good outcomes. Histological examination revealed hyperplasia and not malignancy. The postoperative course was uneventful. The mother and infant were healthy.
\end{abstract}

\section{Keywords}

Compressive Goiter Mother, Obstructed Airway, Pregnancy, Thyroidectomy

\section{Introduction}

Thyroid disease is common in pregnancy, with pre-existing hyper- or hypothyroidism being present in up to $4 \%$ of pregnancies. Indeed, owing to the structural analogy of the $\alpha$ subunit of $\beta$ hCG to thyroid stimulating hormone, production of T4 and T3 is greatly enhanced. This enhanced thyroid stimulation results in relative hypothyroxinemia in women with insufficient dietary Iodine $(<200 \mu \mathrm{g}$ per day) resulting in thyroid gland hypertrophy (goitrogenesis) of up to $20 \%$ to $30 \%$ increased volume during pregnancy, which may only partially regress after childbirth [1]. In this case report, we describe the successful management of a pregnant woman with severe thyroid disease.

\section{Case Description}

A 27-year-old G4P3 woman presented acutely at 37 weeks gestation with increasing respiratory distress and stridor. Over the past two years, the patient described a progressively enlarging mass within her neck accompanied by worsen- 
ing exercise tolerance, dysphagia, and orthopnea. We note that aside from a BMI of 36, this woman has no other medical comorbidities, has never smoked, and has had 3 previous normal vaginal deliveries with uncomplicated pregnancies.

A neck ultrasound performed 12 months prior revealed a significant goiter which even at that point in time showed tracheal deviation and 3 nodules concerning for malignancy. Unfortunately, specialist review with the regional ENT (ear, nose and throat) service was delayed, and her first specialty assessment was at 6 weeks gestation of the index pregnancy. At that time, thyroid function tests and nasolaryngoscopy were performed and found to be normal while confirming the presence of an enlarged left lobe of the thyroid. Fine needle aspiration of the 3 concerning nodules were reported as benign. At that stage, a total thyroidectomy and bilateral tonsillectomy to alleviate her compressive symptoms was planned to be performed postnatally.

However, the goiter continued to enlarge, and became increasingly symptomatic such that by the time of her acute presentation, she had minimal exercise tolerance with such severe orthopnea that she was unable to sleep nor maintain her oxygen saturation without remaining completely upright. This condition was progressive and not associated with any acute systemic illness. On examination, the goiter was again noted, with a clearly audible inspiratory stridor. While her Oxygen Saturation whilst upright was $97 \%$ on air with a respiratory rate of 20 , her respiratory status deteriorated rapidly with marked hypoxemia with only minimal movement towards a supine position. Fetal monitoring was reassuring.

On imaging (Xray (Figure 1) and CT (Figure 2, Figure 3)) retrosternal extension of the left lobe of the thyroid was demonstrated producing tracheal deviation to the right, and compression of the trachea down to $5 \mathrm{~mm}$ diameter at its smallest. Aside from iron deficiency anemia, all blood work was grossly normal including thyroid function tests showing euthyroidism.

After a multidisciplinary discussion involving local endocrine surgeons, ENT specialists, anesthesiologists, and obstetricians, a plan was made to perform a

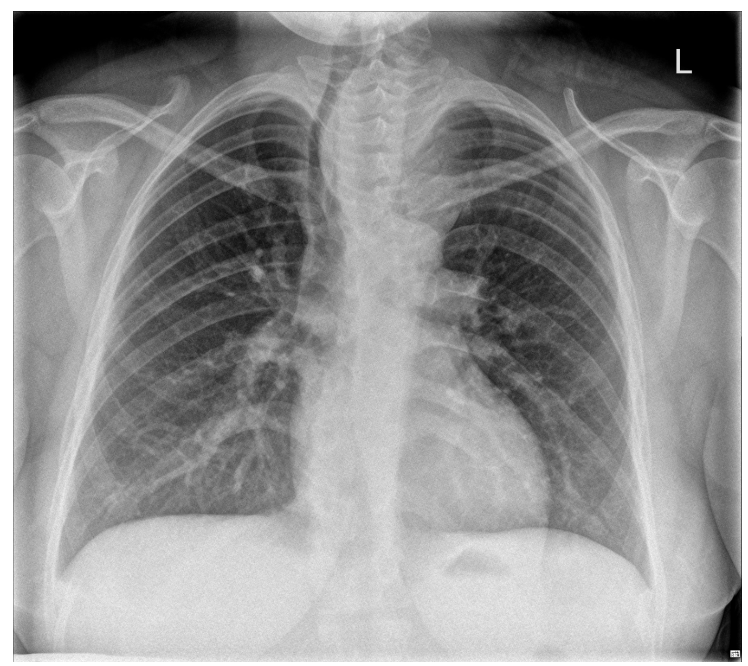

Figure 1. Chest X-Ray showing a deviated trachea. 


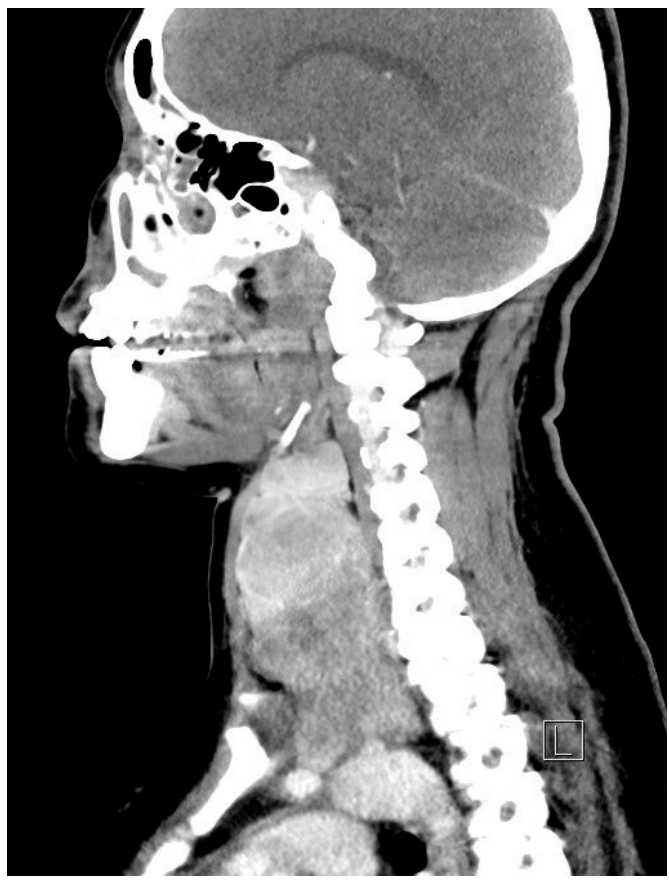

Figure 2. CT: sagittal view of goiter.

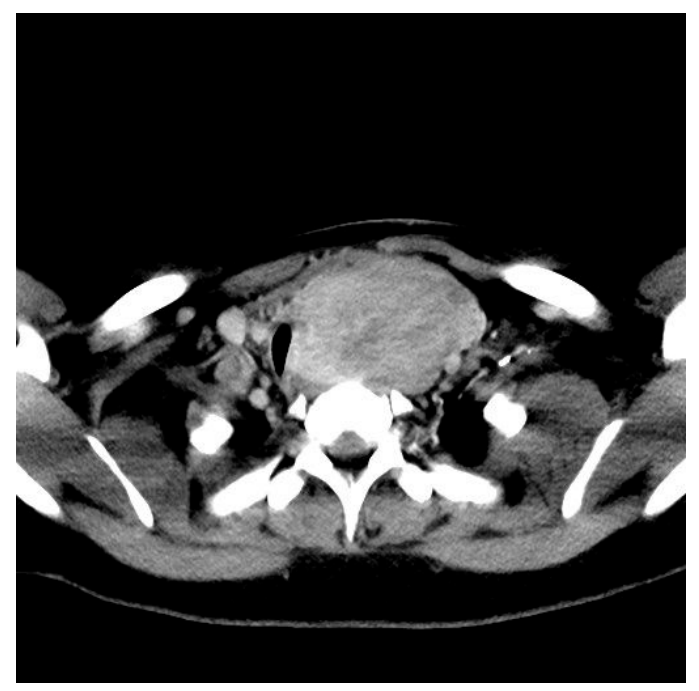

Figure 3. CT: transverse view of goiter and deviated trachea, with a diameter of $5 \mathrm{~mm}$ at smallest point.

combined procedure involving caesarean section followed by total thyroidectomy under general anesthesia. This decision took into account the fact that the pregnancy was term, the woman had a low likelihood of tolerating labor, due to the tracheal compression producing severe respiratory compromise even at rest. Given the risk of this procedure and the reassuring observations of this patient, although clinically in distress, the operation was undertaken the next day when full staffing was available, whilst the patient was admitted overnight to the High Dependency Unit (HDU) for monitoring.

The surgery was overall uncomplicated; after the intubation, a baby girl of 2.8 
$\mathrm{kg}$ was born via caesarean section followed by a difficult but uncomplicated total thyroidectomy with reimplantation of the left superior parathyroid in the left sternocleidomastoid muscle. Postoperatively, the patient was again admitted to HDU as precaution. However, she made an uneventful recovery and she and her baby were discharged after 4 days with prescription for lifelong Levothyroxine $100 \mu \mathrm{g}$ and calcitriol and calcium tablets for 2 weeks. Blood results including electrolytes and calcium were normal at discharge and a plan was made for ongoing endocrine monitoring through her primary care provider.

Histology confirmed an asymmetric thyroid with the right gland weighing 20 $\mathrm{g}$ and containing a $2 \mathrm{~mm}$ foci of noninvasive follicular thyroid neoplasm, but no signs of malignancy, and the left lobe (Figure 4) weighing $186 \mathrm{~g}$ with nodular hyperplasia but no signs of atypia and malignancy. One parathyroid gland was also noted.

\section{Discussion}

This patient had an enlarged thyroid, weighing more than 40 grams, resulting in dyspnea and dysphagia from compression of the trachea, larynx and esophagus. When treatment is delayed, the thyroid gland can extend retrosternally or into adjacent areas of the neck [2] [3]. Treatment of goiters, after exclusion of malignancy or suspicious nodules, depends on the symptoms. Asymptomatic patients may be observed or can be treated with radioactive iodine. Regular monitoring is indicated. If dysphagia, choking sensations, or airway obstruction are present, surgery is necessary to alleviate these symptoms [4].

Risks associated with thyroid surgery include unintentional resection of parathyroid glands and injury to the recurrent laryngeal nerve. Resection of the parathyroid glands can lead to hypoparathyroidism and hypocalcemia, which is characterized by facial muscle spasms, muscle cramps, paresthesia of lips, tongue, fingers and feet, tetany and seizures. Furthermore, in a chronic setting, this can lead to a fetus with skeletal demineralization resulting in multiple bone fractures in the neonatal period [5]. The consequence of maternal hypothyroidism,

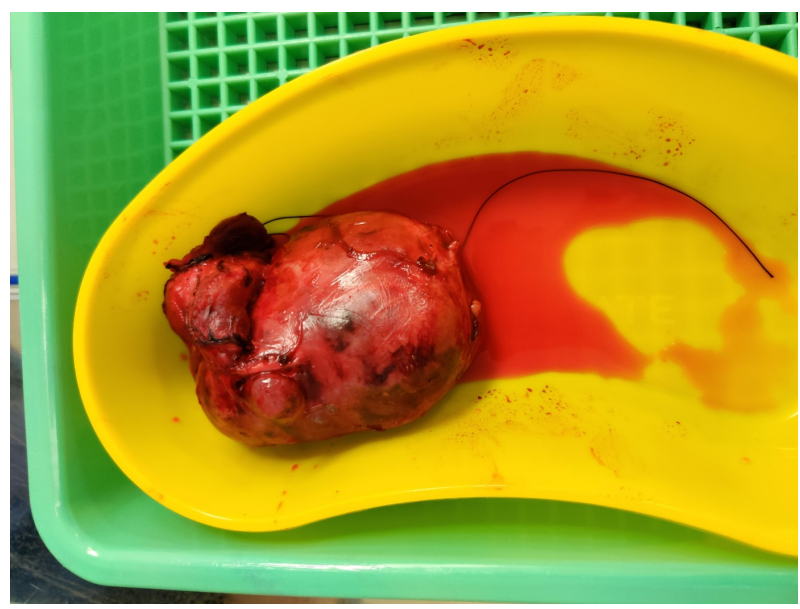

Figure 4. Left thyroid lobe. 
such as impaired neurological and physical development, should be closely monitored and levothyroxine dosing adjusted accordingly. Besides surgical complications, other risks associated with surgery in pregnancy are also present such as altered organogenesis and spontaneous abortion if surgery is performed in the first trimester, alongside preterm labor and delivery if performed in the third trimester. In situations where surgery in pregnancy is indicated, it should ideally be performed in the second trimester to minimize the risks for mother and fetus [6]. However where possible, surgery should be deferred until after delivery [7].

The largest retrospective study comparing thyroid surgery in pregnant and non-pregnant women was conducted by Kuy et al. in 2009 [8]. Pregnant patients were twice as likely to have surgical complications than similar nonpregnant women, ended up staying longer in hospital and had higher hospital costs. The fetal and maternal complication rates were $5.5 \%$ and $4.5 \%$ respectively. However, a substantial baseline difference was present as pregnant women were more likely to have urgent admission and higher percentage of government insurance, the latter being also an independent predictor of outcome. Other explanations for the higher complication rate may be the increase of thyroid volume in pregnancy and more advanced thyroid disease at time of the operation, contributing to operative difficulty.

Earlier case reports describe different outcomes, varying from an emergency thyroidectomy at 32 weeks after respiratory arrest followed by a normal delivery at 37 weeks [9], and a thyroidectomy at 34 weeks followed by a normal delivery at 40 weeks [10], to an elective caesarean section at 32 weeks with planned thyroidectomy 4 weeks later [11]. These various outcomes illustrate the complexity of management of these patients and show the need for a multidisciplinary plan.

\section{Conclusion}

While thyroid disease is common in pregnancy, a life-threatening obstructive goiter is not. These patients need a team-based management plan, as early on in pregnancy as possible, taking into account the severity of the disease, the symptoms, if present, the malignancy risk and the maternal and fetal risks. If an elective thyroidectomy is necessary, we believe this is best planned in the second trimester to prevent adverse outcomes further in pregnancy.

\section{Consent}

Written consent of the patient was obtained before publication.

\section{Conflicts of Interest}

The authors declare no conflicts of interest regarding the publication of this paper.

\section{References}

[1] Glinoer, D. (1999) What Happens to the Normal Thyroid during Pregnancy? Thy- 
roid, 9, 631-635. https://doi.org/10.1089/thy.1999.9.631

[2] White, M.L., Doherty, G.M. and Gauger, P.G. (2008) Evidence-Based Surgical Management of Substernal Goiter. World Journal of Surgery, 32, 1285-1300.

https://doi.org/10.1007/s00268-008-9466-3

[3] Thomas, C.M., Mattingly, J.K., Hendrickse, A. and Song, J.I. (2017) Case Report of a Massive Retropharyngeal Goiter Resulting in Laryngeal Compression. A \& A Case Reports, 9, 178-181. https://doi.org/10.1213/XAA.0000000000000560

[4] Bahn, R.S. and Castro, M.R. (2011) Approach to the Patient with Nontoxic Multinodular Goiter. The Journal of Clinical Endocrinology \& Metabolism, 96, 1202-1212. https://doi.org/10.1210/jc.2010-2583

[5] Cunning, F.G., Leveno, K.J., Bloom, S.L., Spong, C.Y., et al. (2014) Williams Obstetrics. 24th Edition, Thyroid Disorders, Chapter 58, McGraw-Hill Education/Medical, New York, 2167-2187.

[6] Stagnaro-Green, A., Abalovich, M., Alexander, E., et al. (2011) Guidelines of the American Thyroid Association for the Diagnosis and Management of Thyroid Disease during Pregnancy and Postpartum. Thyroid, 21, 1081-1125. https://doi.org/10.1089/thy.2011.0087

[7] Ruan, D. and Moalem, J. (2009) Thyroid Gland Pregnancy-A Risk Factor for Thyroid Surgery Complications. Nature Reviews Endocrinology, 5, 589-590. https://doi.org/10.1038/nrendo.2009.211

[8] Kuy, S.R., Roman, S.A., Desai, R. and Sosa, J.A. (2009) Outcomes Following Thyroid and Parathyroid Surgery in Pregnant Women. Archives of Surgery, 144, 399-406. https://doi.org/10.1001/archsurg.2009.48

[9] El Jaouhari, S.D., Doghmi, N., Najout, H., et al. (2019) Acute Respiratory Failure Secondary to a Cervical Goitre in a Pregnant Woman: A Case Report. BMC Emergency Medicine, 19, 18. https://doi.org/10.1186/s12873-019-0231-8

[10] Ridal, M., Abbasi, H., Aissaoui, F. and Alami, M.N. (2015) La priseen charge d'un goiter compressif chez une femme enceinte [Management of a Compressive Goiter in a Pregnant Woman]. Tunisie Medicale, 93, 187-189.

[11] Al-Shammari, L., Jemmett, K., Wikner, M. and Usman, N. (2015) Management of a Parturient with a Retrosternal Goitre and Tracheal Compression. International Journal of Obstetric Anesthesia, 24, 201-202. https://doi.org/10.1016/j.ijoa.2015.02.004 